\title{
Exploring the Potential of Multi-Detector Analyses for Core-Collapse Supernova Neutrino Detection
}

\author{
Meriem Bendahman, ${ }^{a, b}$ Matteo Bugli, ${ }^{c}$ Alexis Coleiro, ${ }^{b}$ Marta Colomer Molla, ${ }^{e}$ \\ Gwenhaël de Wasseige, ${ }^{b}$ Thierry Foglizzo, ${ }^{c}$ Antoine Kouchner, ${ }^{b, d}$ Mathias Regnier, ${ }^{b}$ \\ Yahya Tayalati, ${ }^{a}$ Alessandra Tonazzo ${ }^{b}$ and Véronique Van Elewyck ${ }^{b, d}$ \\ ${ }^{a}$ Faculty of Sciences, Mohammed V University, Rabat, Morocco \\ ${ }^{b}$ Université de Paris, CNRS, Astroparticule et Cosmologie, Paris, France \\ ${ }^{c}$ CEA Saclay, Department of Astrophysics, Gif-sur-Yvette, France \\ ${ }^{d}$ Institut Universitaire de France, 1 rue Descartes, Paris, France \\ ${ }^{e}$ Inter-university Institute for High Energies, Université libre de Bruxelles (ULB), Brussels, Belgium \\ E-mail: mbendahman@km3net.de
}

The core-collapse supernova (CCSN) SN1987A has been the first extragalactic transient source observed with neutrinos, and the 25 events detected by the neutrino telescopes running at that time marked the beginning of neutrino astronomy. Despite hundreds of CCSNs detected in the electromagnetic spectrum since 1987, neutrino telescopes could not perform another observation due to the far distances of the sources. It is thus of primary importance to optimize the detection channel of sensitive detectors in anticipation of a forthcoming Galactic CCSN. Beyond being used as an early warning of a close-by CCSN, neutrinos can provide unique information on the explosion mechanism, and can be used to probe neutrino oscillation in dense environments. In this contribution, we present the potential of multi-detector analysis to enhance the scientific outputs from the next close-by CCSN. Combining the expected light curves in neutrino detectors sensitive to different flavors, we study the constraints that could be set on the properties of the progenitor itself, such as its mass, as well as on the neutrino oscillation parameters. We also present the results of a triangulation algorithm using a prior source map in the definition of the the region of interest.

$37^{\text {th }}$ International Cosmic Ray Conference (ICRC 2021)

July 12 th - 23rd, 2021

Online - Berlin, Germany 


\section{State of the art and Motivation}

In the era of multi-messenger astronomy, the transient sky holds a place of special importance for the community. Nowadays, high-energy neutrino telescopes systematically provide upper limits on the neutrino flux emitted during remarkable transient events such as compact binary mergers or gamma-ray bursts [1]. Contrariwise, the $\mathrm{MeV}-\mathrm{GeV}$ regime has been poorly studied so far and only a few constraints have been set by e.g., Super-Kamiokande [2] and IceCube [3]. The only transient source that has been observed in this low-energy range so far is the core-collapse supernova (CCSN) SN1987A, which led to 25 neutrino events detected in Kamiokande, the Irvine-MichiganBrookhaven (IMB) and the Baksan Neutrino Observatory [4]. This unique detection marked the beginning of neutrino astronomy and allowed to confirm the so-called baseline model "delayed explosion mechanism". Despite hundreds of CCSNs detected in the electromagnetic spectrum since 1987, neutrino telescopes could not perform another observation due to the far distances of these events. It is thus of primary importance to optimize the detection channel of sensitive detectors in anticipation of a forthcoming CCSN. Beyond being used as an early warning of a galactic CCSN, neutrinos can provide unique information on the explosion mechanism, and can be used to probe neutrino oscillations in dense environments [5]. In this contribution, we study different techniques to enhance the potential of neutrino telescopes to low-energy astrophysical neutrinos. We start by briefly presenting the various instruments we have used for this work. Section 3 investigates the potential of using a Bayesian approach to triangulate the position of a close-by CCSN. In Section 4, we explore the potential of multi-detector analyses in constraining the characteristics of the CCSN as well as neutrino oscillation parameters. We conclude with the prospects for similar analyses.

\section{Description of the experiments}

This section briefly introduces the different experiments used in the presented work. Table 1 summarizes the main characteristics of each detector and the estimated number of detected events from a CCSN at $10 \mathrm{kpc}$ for each of them.

DarkSide is a collaboration planning to build and operate a series of liquid argon time projection chambers (TPCs) that are employed at the Gran Sasso National Laboratory in Assergi in Italy. The main goal of DarkSide is the direct detection of dark matter and it is sensitive to the recoil of the nuclei after collisions with dark matter particles or neutrinos. [9].

DUNE (Deep Underground Neutrino Experiment) will consist of two neutrino detectors. One will record particle interactions near the beam source, at the Fermi National Accelerator Laboratory in Batavia, Illinois. The second one will consist of 4 detection volumes and will be installed more than a kilometer underground at the Sanford Underground Research Laboratory in Lead, South Dakota, 1300 kilometers downstream from the source. These detectors will be dedicated to the study of neutrino physics and their role in the universe [8].

IceCube is a neutrino observatory built at the South Pole, made of 5160 digital optical modules located in the Antarctic ice between 1450 and $2450 \mathrm{~m}$ below the surface and spread over a cubic kilometer. Each module contains a photo-multiplier tube and an acquisition card to transfer digital data to the station. IceCube has been primarily optimized to search for high-energy astrophysical neutrinos [6]. 
KM3NeT (Kilometer Cube Neutrino Telescope) is an underwater telescope under construction in the Mediterranean Sea. It is being deployed at two different sites: ORCA (Oscillation Research with Cosmics in the Abyss) is dedicated to the study of neutrino properties, while ARCA (Astroparticle Research with Cosmics in the Abyss) is used for the observation of cosmic neutrinos. These two volumes are instrumented with digital optical modules that are associated in vertical groups of 18 to form detection lines. In total, 115 of these lines are planned to form the ORCA detector and 230 lines will be used to create ARCA [7].

Super-Kamiokande is a water Cherenkov detector located $1000 \mathrm{~m}$ underground containing 50,000 tons of pure water in the Kamioka zinc mine in the Gifu prefecture of Japan. The detector consists of an inner detector and an outer veto detector, both are contained within a cylindrical stainless steel tank $39.3 \mathrm{~m}$ in diameter and $41.4 \mathrm{~m}$ in height. The outer detector is a water Cherenkov detector of 13000 metric tons total mass [2].

These detectors are part of the SNEWS 2.0 Collaboration [10]. SNEWS 2.0 is an upgraded version

Table 1: Detector characteristics and total number of expected events assuming normal (left) and inverted (right) mass ordering for a $27 \mathrm{M}_{\odot}$ and $11 \mathrm{M}_{\odot} \mathrm{CCSN}$ at $10 \mathrm{kpc}$ given in [10].

\begin{tabular}{|c|c|c|c|c|}
\hline \multirow{2}{*}{ Experiment } & Detected & Effective masse & \multicolumn{2}{|c|}{ Estimated number of events } \\
& neutrino flavour & $\mathbf{( k T )}$ & $\mathbf{1 1 . 2} \mathbf{M}_{\odot}$ & $\mathbf{2 7 ~ M}_{\odot}$ \\
\hline KM3NeT & $\bar{v}_{e}$ & 150 & $17 \times 10^{3} / 18 \times 10^{3}$ & $37 \times 10^{3} / 38 \times 10^{3}$ \\
\hline DUNE & $v_{e}$ & 40 & $2700 / 2500$ & $5500 / 5200$ \\
\hline DarkSide-20K & any $v$ & 0.0386 & - & 250 \\
\hline IceCube & $\bar{v}_{e}$ & 2500 & $320 \times 10^{3} / 330 \times 10^{3}$ & $660 \times 10^{3} / 660 \times 10^{3}$ \\
\hline Super-Kamiokande & $\bar{v}_{e}$ & 32 & $4000 / 4100$ & $7800 / 7600$ \\
\hline
\end{tabular}

of SNEWS system that exploits the advantages of rapid neutrino detection to study the science from these events. The main goal of SNEWS is to combine data from different neutrino observatories, and provide summaries of neutrino data. SNEWS 2.0 enables the integration of dark matter detectors as inputs to SNEWS, in addition to the detectors that were already part of the SNEWS system. Another addition is the combination of coherent elastic neutrino-nucleon scattering (CE $v \mathrm{NS}$ ) and inelastic interaction channels to study oscillation effects. SNEWS 2.0 will also intend to develop and provide a true pre-supernova alert [10].

\section{Multi-detector approach for CCSN triangulation}

Several efforts have already been carried out to triangulate the position of the CCSN using neutrino telescope data. We hereby use the approach described in [11], which estimates the time delay between the light curves recorded by different detectors during a CCSN to infer its position in the sky. In this study, we have used detectors sensitive to the same flavor, i.e. IceCube, KM3NeT, and Super-Kamiokande, to ensure that any delay in the recorded light curves would come from geometrical effects. The time difference between the CCSN neutrino signal arrival at two detectors located at $\vec{r}_{i}$ and $\vec{r}_{j}$ can be expressed as:

$$
t_{i j}=\left(\vec{r}_{i}-\vec{r}_{j}\right) \cdot \vec{n} / c
$$

where $\vec{n}$ is the unit vector that indicates the incoming neutrino direction. It is calculated in the 
geographic horizontal coordinate system from the CCSN right ascension, $\alpha$, declination, $\delta$, and the Greenwich mean sidereal time expressed as angle, $\gamma$ :

$$
\vec{n}=(-\cos (\alpha-\gamma) \cos \delta,-\sin (\alpha-\gamma) \cos \delta,-\sin \delta) .
$$

The position of the detector $k$ can be inferred from its latitude, $\phi_{k}$, and longitude, $\lambda_{k}$, angles, and the Earth radius, $R_{\text {Earth }}$ :

$$
\overrightarrow{r_{k}}=R_{E \operatorname{arth}}\left(\cos \lambda_{k} \cos \phi_{k}, \sin \lambda_{k} \cos \phi_{k}, \sin \phi_{k}\right) .
$$

The probability that the scanned angles $(\alpha, \delta)$ coincide with the equatorial coordinates of the CCSN is given by the following $\chi^{2}$ function:

$$
\chi_{i j}^{2}(\alpha, \delta)=\left(\left(t_{i j}(\alpha, \delta)-T_{0, i j}^{m a t c h}\right) / \delta t_{i j}\right)^{2}
$$

with no systematic shift in the $T_{0, i j}^{\text {match }}$ determination. The best estimate for the angles $(\alpha, \delta)$ of the searched CCSN location in the sky is given by the minimum of the function. The performance depends on the uncertainty of the measured time delay $\delta t_{i j}$ of each detector pair.

To further develop this approach, we studied the impact of using a prior on the position of the potential CCSN through a Bayesian approach. The tested prior was a map of GAIA showing the star density distribution in the Milky Way. This approach allows us to reduce the $90 \%$ confidence area of the source localization by more than $55 \%$, depending on the combination of neutrino telescopes which is used, as shown in Fig 1.

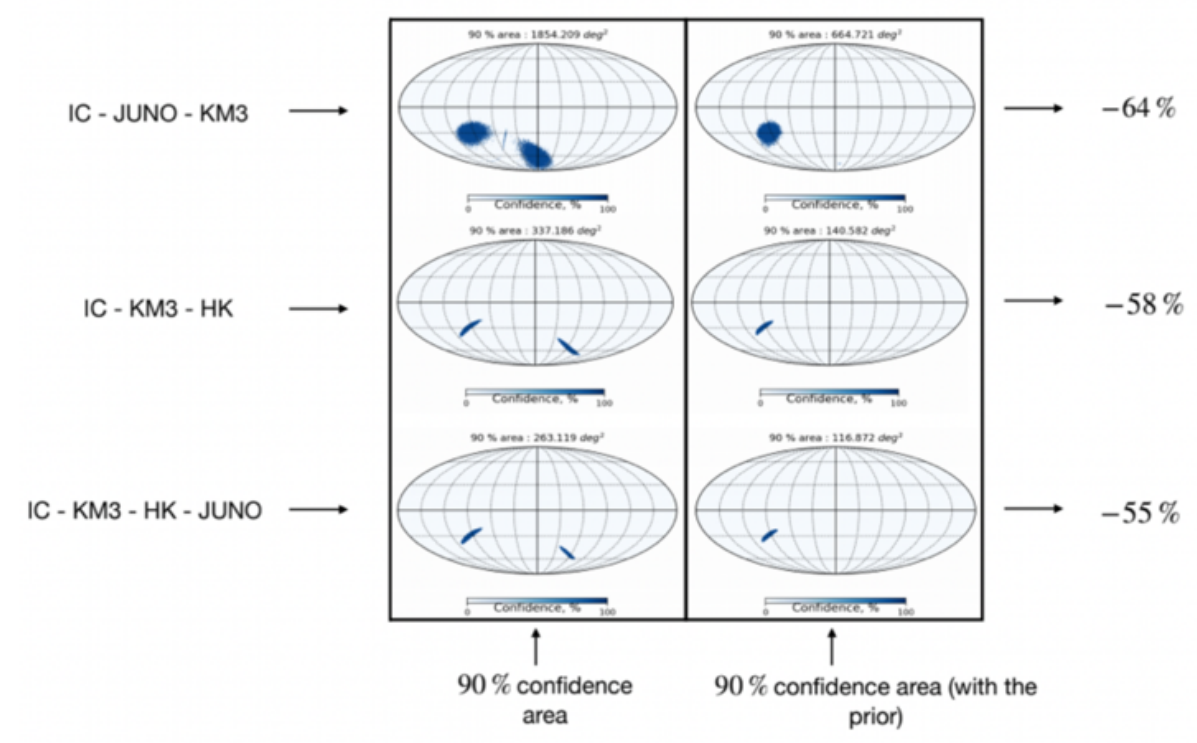

Figure 1: Comparison of the confidence areas obtained by the CCSN triangulation method [11] with and without using the prior for a CCSN located at the Galactic Center.

\section{Multi-detector approach for enhancing the scientific output}

In this section, we describe the combination of the predicted light curves seen in KM3NeT, DUNE, and DarkSide-20k. Each of these detectors is predominantly sensitive to a different neutrino flavor. The combination of the recorded light curves may provide a global view on the neutrino emission during the different phases of the CCSN. The goal of this study is to use variables such as ratios or asymmetries between the number of events that would be detected in these three detectors 

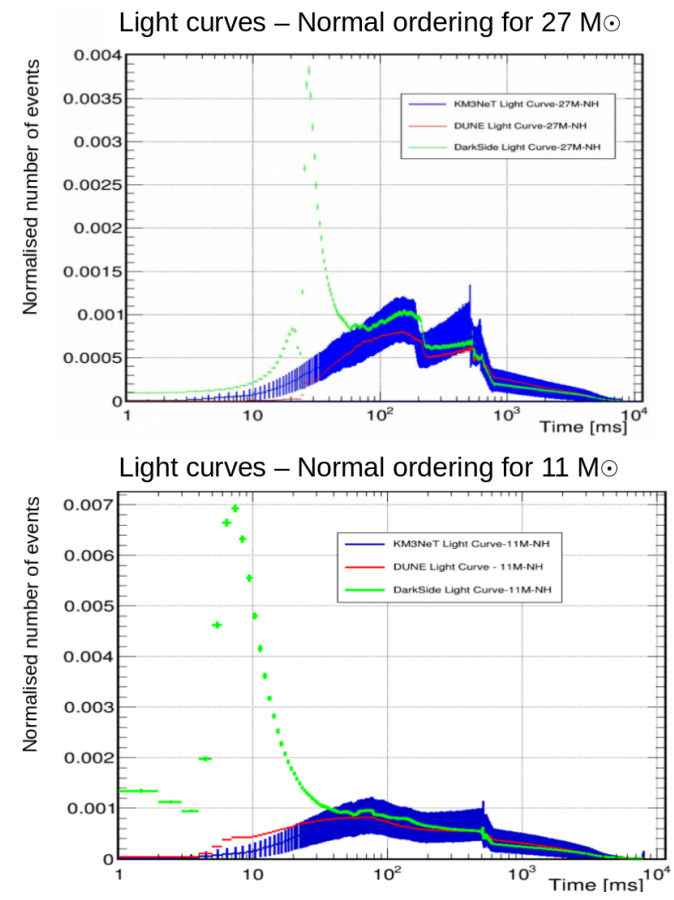

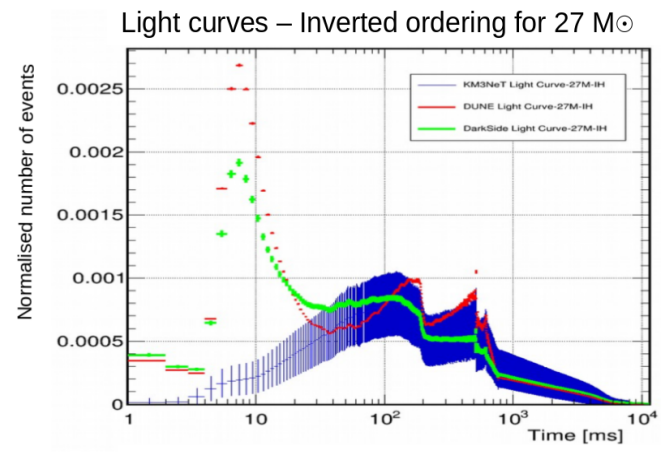

Light curves - Inverted ordering for $11 \mathrm{M} \odot$

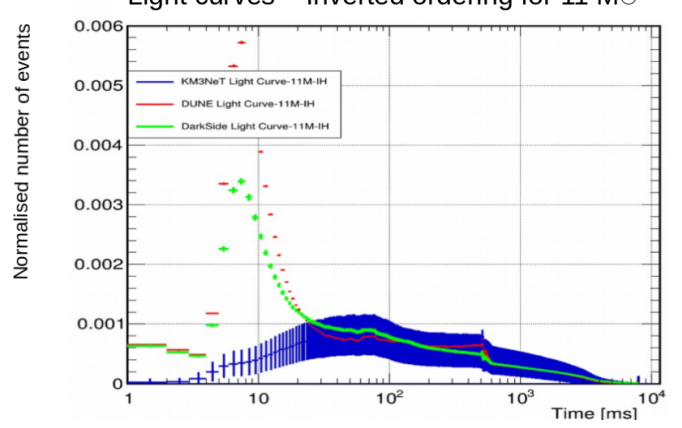

Figure 2: Normalized light curves from KM3NeT, DUNE and DarkSide respectively in blue, green and red for $27 \mathrm{M}_{\odot}$ (top), $11 \mathrm{M}_{\odot}$ (bottom), normal ordering (left) and inverted ordering (right).

to determine the neutrino mass ordering and estimate the mass of the progenitor. The different hypotheses will influence the number of neutrinos detected as a function of time for the various instruments, as shown in Fig. 2.

Light curve comparison We start by deriving the expected fluxes for each neutrino flavor using the CCSN simulation models for the $27 \mathrm{M}_{\odot}$ and $11 \mathrm{M}_{\odot}$ progenitors from [12], and we then compute the corresponding number of events expected to be observed in each detector. The event rate can be estimated as the product of the differential neutrino flux $\frac{d \Phi}{d E_{v}}$, the cross section $\sigma$ of each relevant interaction channel, the detection efficiency $\epsilon_{d e t}$, and the detector volume $V_{d e t}$ :

$$
N_{e v}=\int_{E \min }^{E \max } d E \frac{d \Phi}{d E_{v}} \sigma \epsilon_{\text {det }} V_{\text {det }} .
$$

For DUNE, the cross section used is from the Marley model from SNOwGLoBES [13]: the main considered interactions are $v_{e}$ charged current interactions on Argon, $\bar{v}_{e}$ charged current interactions and elastic scattering on electrons for all flavor are also considered. We assume $100 \%$ efficiency above $5 \mathrm{MeV}$. In the case of DarkSide-20k, the effective mass is of 49.7 tons, the considered cross section is the one of CE $v$ NS in addition to the same interactions as in DUNE, and $100 \%$ efficiency is assumed for the detector in the full energy range. For KM3NeT, we first calculate the interaction rate in $100 \mathrm{kt}$ on water considering inverse beta decay, elastic scattering for all flavors, and interactions with oxygen nuclei as main interaction channels. The effective mass and the energy dependent efficiency parametrisation that were build for SNOWGLOBES are used [13]. Two studies were carried out in this analysis: we investigated the impact of the neutrino mass ordering on the 

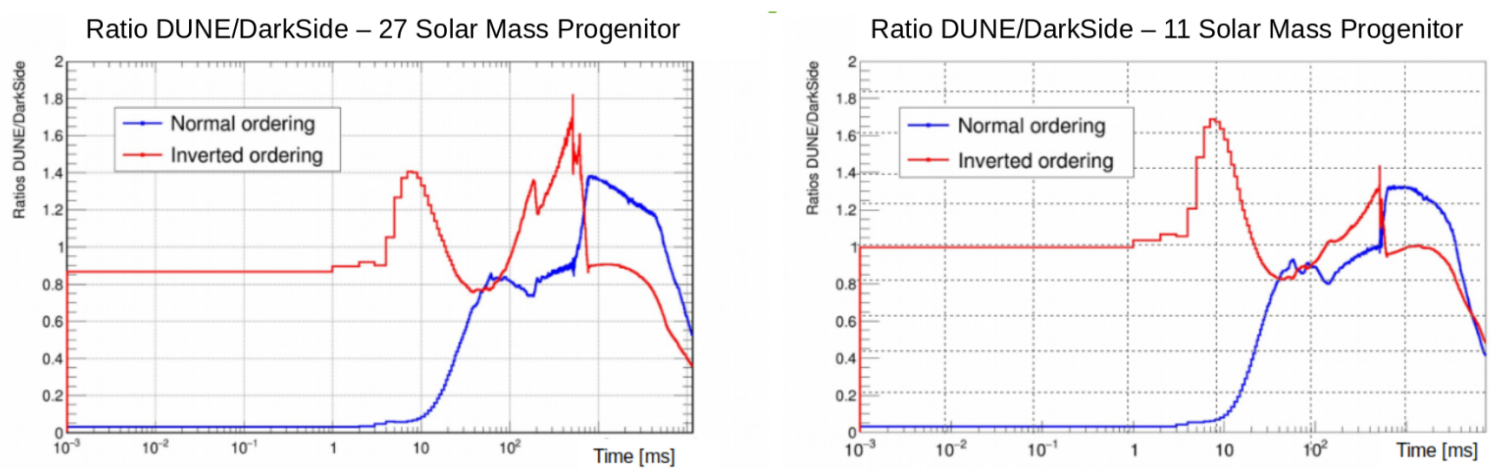

Figure 3: Ratios $\left(\mathrm{N}_{\operatorname{det} 1}, \mathrm{~N}_{\mathrm{det} 2}\right)=(\mathrm{DUNE}$, DarkSide) for normal (blue) and inverted (red) mass ordering for $27 \mathrm{M}_{\odot}($ left $)$ and for $11 \mathrm{M}_{\odot}$ (right).

neutrino light curve produced by a $27 \mathrm{M}_{\odot}$ and a $11 \mathrm{M}_{\odot}$ progenitors, and we studied the difference between these mass progenitors assuming both normal and inverted neutrino mass ordering. To compare the light curves recorded by the different detectors shown in Fig 2, we computed the ratio and the asymmetry between the light curves as follow:

$$
\text { Ratio }=\mathrm{N}_{\operatorname{det} 1} / \mathrm{N}_{\mathrm{det} 2} \text { and Asymmetry }=\left(\mathrm{N}_{\operatorname{det} 2}-\mathrm{N}_{\operatorname{det} 1}\right) /\left(\mathrm{N}_{\mathrm{det} 2}+\mathrm{N}_{\operatorname{det} 1}\right),
$$

where $\mathrm{N}_{\mathrm{det} 1}$ represents the number of events in one of the detector integrated over the considered time window, and $\mathrm{N}_{\operatorname{det} 2}$ is the number of events in another detector during the same time window. We considered the following combinations : $\left(\mathrm{N}_{\operatorname{det} 1}, \mathrm{~N}_{\mathrm{det} 2}\right)=($ DUNE, DarkSide $)$ and $\left(\mathrm{N}_{\operatorname{det} 1}, \mathrm{~N}_{\mathrm{det} 2}\right)$ $=(\mathrm{KM} 3 \mathrm{NeT}+\mathrm{DUNE}$, DarkSide $)$.

Determination of the neutrino mass ordering In this section, the ratios $\left(\mathrm{N}_{\operatorname{det} 1}, \mathrm{~N}_{\mathrm{det} 2}\right)=(\mathrm{DUNE}$, DarkSide) are shown in Fig 3 for each neutrino mass ordering assuming a $27 \mathrm{M}_{\odot}$ and a 11 $\mathrm{M}_{\odot}$ progenitors, respectively. Similar results have been obtained for the ratio $\left(\mathrm{N}_{\operatorname{det} 1}, \mathrm{~N}_{\operatorname{det} 2}\right)=$ (KM3NeT+DUNE, DarkSide). Both the ratio and the asymmetry for the two considered detector combinations show significant differences around the neutronization peak.

Constraints on the progenitor mass Asymmetries $\left(\mathrm{N}_{\mathrm{det} 1}, \mathrm{~N}_{\mathrm{det} 2}\right)=(\mathrm{KM} 3 \mathrm{NeT}+\mathrm{DUNE}$, DarkSide $)$ are shown in Fig 4 for the determination of the mass of the progenitor assuming normal and inverted mass ordering. Similar results have been obtained for the asymmetry $\left(\mathrm{N}_{\operatorname{det} 1}, \mathrm{~N}_{\operatorname{det} 2}\right)=($ DUNE, DarkSide). In this case, only small differences are found between the two studied progenitor masses. The next section describes how we identified the most sensitive time window to differentiate between the two hypotheses.

Statistical methods for model discrimination In order to find the most promising time window, detector combination, and variable for each hypothesis, i.e. the mass ordering or the mass of the progenitor, we loop over the time throughout the duration of the light curves by step of $10 \mathrm{~ms}$. For each possible time window, we calculate the difference in numbers of sigma between the two hypotheses. The results are show in Fig. 5, where for each time window in milliseconds, the ratio and asymmetry difference between the tested hypotheses have been expressed in number of the 

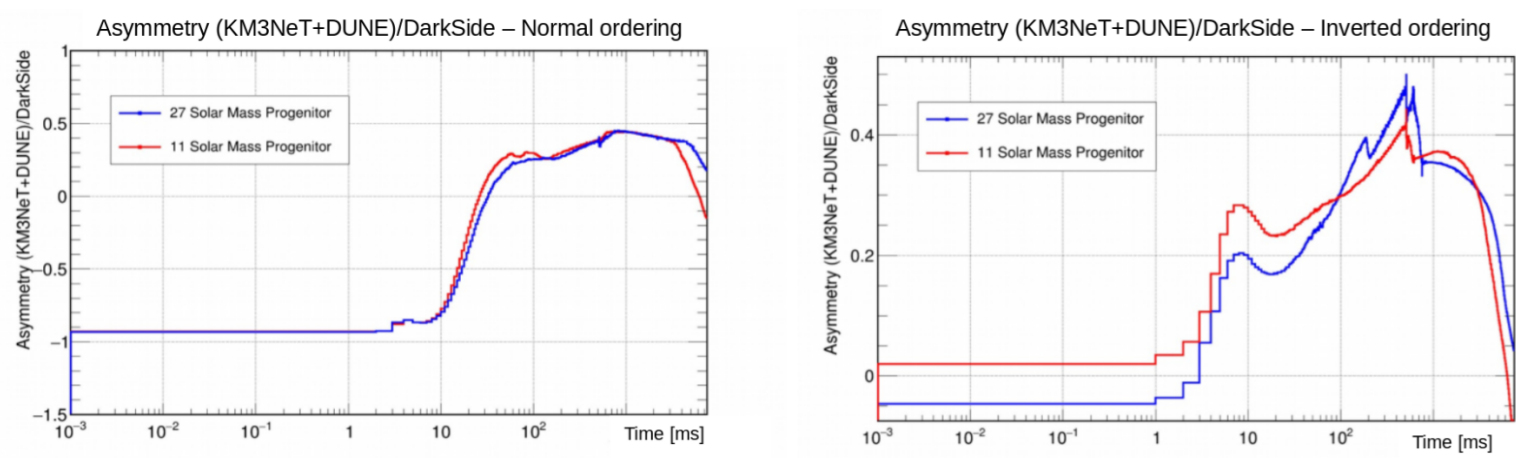

Figure 4: Asymmetries $\left(\mathrm{N}_{\operatorname{det} 1}, \mathrm{~N}_{\operatorname{det} 2}\right)=\left(\mathrm{KM} 3 \mathrm{NeT}+\mathrm{DUNE}\right.$, DarkSide) for $27 \mathrm{M}_{\odot}$ (blue) and $11 \mathrm{M}_{\odot}$ (red) assuming normal (left) and inverted (right) mass ordering.

largest statistical uncertainty $\sigma$. No time window leading to a $3 \sigma$ difference could be identified for the two progenitor masses considered in the study. Concerning the mass ordering study, we find a significant difference between the ratios of DUNE/DarkSide and(KM3NeT+DUNE)/DarkSide of more than $14 \sigma$ for $[1,10] \mathrm{ms}$ and more than $6 \sigma$ for $[1,20] \mathrm{ms}$. The asymmetries between (DUNE, DarkSide) and (KM3NeT+DUNE, DarkSide) also leads to a still significant but smaller difference of more than $3 \sigma$ for the $[1,10]$ ms time window.
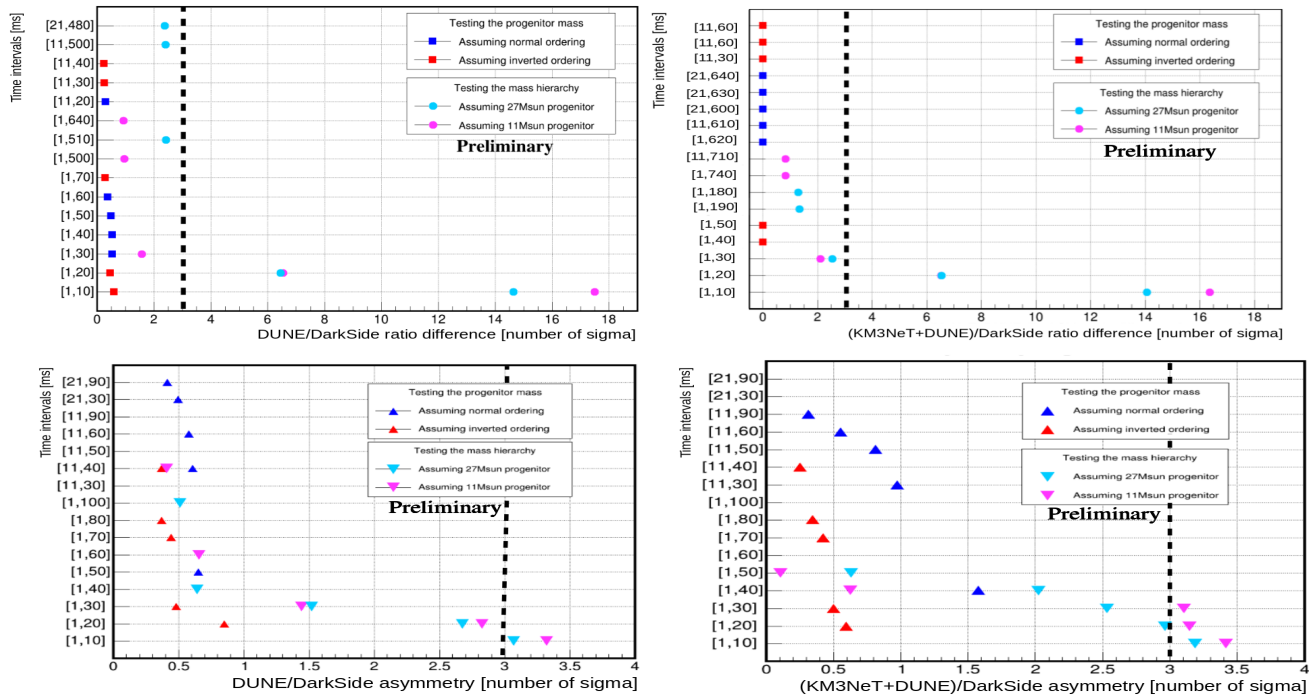

Figure 5: Mass ordering and progenitor mass estimate for the ratio and asymmetry variables.

\section{Summary and Prospects}

In this contribution, we presented some preliminary efforts to carry out multi-detector analyses enhancing the scientific output of the next Galactic CCSN. We first studied the benefit of using a Bayesian approach to include a prior in an existing triangulation method. The proposed method 
leads to reduce the $90 \%$ confidence area of the source localization by more than 55\% for all the considered detector combinations for a CCSN located at the Galactic Center. We then studied the potential of combining detectors sensitive to different neutrino flavors to determine the neutrino mass ordering and constraining the mass of the CCSN progenitor. Using the technique proposed in this contribution, one can determine the neutrino mass ordering with more than $14 \sigma$ for $[1,10] \mathrm{ms}$ when considering the ratios for (DUNE, DarkSide) and (KM3NeT+DUNE, DarkSide). The sensitivity is expected to drop when poissonian noise will be taken into account in KM3NeT, and this impact will be evaluated in the near future. However, we are currently working on a combination of IceCube and $\mathrm{KM} 3 \mathrm{NeT}$ data that may allow large $\bar{v}_{e}$ detectors to play a significant role on our multi-detector analysis when testing different hypotheses.

This work constitutes a prototype of what can be implemented within SNEWS2.0. We have shown that the mass ordering could be determined by using only the integrated number of events recorded during the first $10 \mathrm{~ms}$ of the light curves, which represents a small amount of data to be shared among the experiments. Further studies will be carried out in the same direction, e.g., studying the sensitivity of the proposed technique to different magnetic field topology around the progenitor.

Acknowledgement This work has been made feasible through the financial support of the LabEx UnivEarthS (ANR-10-LABX-0023 and ANR-18-IDEX-0001). G. de Wasseige acknowledges support from the European Union's Horizon 2020 research and innovation programme under the Marie Sklodowska-Curie grant agreement No 844138.

\section{References}

[1] LIGO Scientific and Virgo Collaborations, Phys. Rev. Lett. 119 (2017) 161101.

[2] Super-Kamiokande Collaboration, Astrophys. J., 669 (2007) 519.

[3] IceCube Collaboration, arXiv:2105.13160 [astro-ph.HE] (2021).

[4] K. Scholberg, Ann.Rev.Nucl.Part.Sci. 62 (2012).

[5] L. Wolfenstein, Phys. Rev. D, 17 (1978) 2369.

[6] IceCube Collaboration, JINST 12 (2017) P03012.

[7] KM3NeT Collaboration, J.Phys. 43 (2012).

[8] DUNE Collaboration, FERMILAB-DESIGN, (2018).

[9] DarkSide Collaboration, arXiv:1802.07198v3 [astro-ph.CO] (2018)

[10] SNEWS Collaboration, New J. Phys. 23 (2021).

[11] A. Coleiro et al., Eur. Phys. J. C 80 (2020) 856.

[12] A. Mirizzi, String Theory 39 (2016) 1.

[13] S. Gardiner, arXiv:2101.11867v1 [nucl-th] (2021). 